\title{
Instantaneous Power Theory For Unbalanced Voltage Compensation Of 3-Phase Power Systems
}

\author{
Gaddam Siva $^{a}$, Dr. Prabodh Kumar Khampariya ${ }^{\text {b }}$ \\ ${ }^{a}$ Research Scholar, Dept. of Electrical Engineering, \\ Sri Satya Sai University of Technology \& Medical Sciences, Sehore, Bhopal Indore Road, Madhya Pradesh, India \\ ${ }^{\mathbf{b}}$ Research Guide, Dept. of Electrical Engineering, \\ Sri Satya Sai University of Technology \& Medical Sciences, Sehore, Bhopal Indore Road, Madhya Pradesh, India
}

Article History: Received: 11 January 2021; Accepted: 27 February 2021; Published online: 5 April 2021

\begin{abstract}
This paper presents another control technique for a three-phase power systems which depends on instantaneous power hypothesis for unbalanced voltage. In this paper we will dissect power quality issues under distortional and unbalanced voltage conditions, In this methodology, the voltage and source voltages are utilized to create the reference voltages of an arrangement dynamic power channel and source currents are utilized to produce the reference currents of a shunt dynamic power channel. The proposed control approach depends on instantaneous power and is streamlined by utilizing a self-tuning channel, without utilizing any filters or phase bolted circle, and without estimating voltage or channel currents. The presentation of the proposed control approach is assessed regarding power factor adjustment, source impartial current alleviation, voltage adjusting and relief of the current and voltage harmonics of distortional and unbalanced voltages in a threephase system. Accordingly, the occasions that current is estimated is diminished and system execution is improved. The outcomes got by MATLAB/SIMULINK programming show the viability of the proposed control procedure in contrast with the regular technique.
\end{abstract}

Keywords: Three phase, power system, voltage, current, instantaneous, unbalanced.

\section{Introduction}

Three-phase electric power is a typical technique for electric power transmission. It is a sort of polyphaser system principally used to power engines and numerous different gadgets. A three-phase system utilizes less conduit material to communicate electric power than identical single-phase, two-phase, or direct-current systems at a similar voltage. Tree-phase system, three circuit conductors convey three alternating currents (of a similar frequency) which arrive at their instantaneous pinnacle esteems at various occasions. Accepting one channel as the reference, the other two currents are postponed in time by $33 \%$ and $66 \%$ of one pattern of the electrical current. This postponement between "phases" has the impact of giving steady power move over each pattern of the current, and furthermore makes it conceivable to create a turning attractive field in an electric engine.
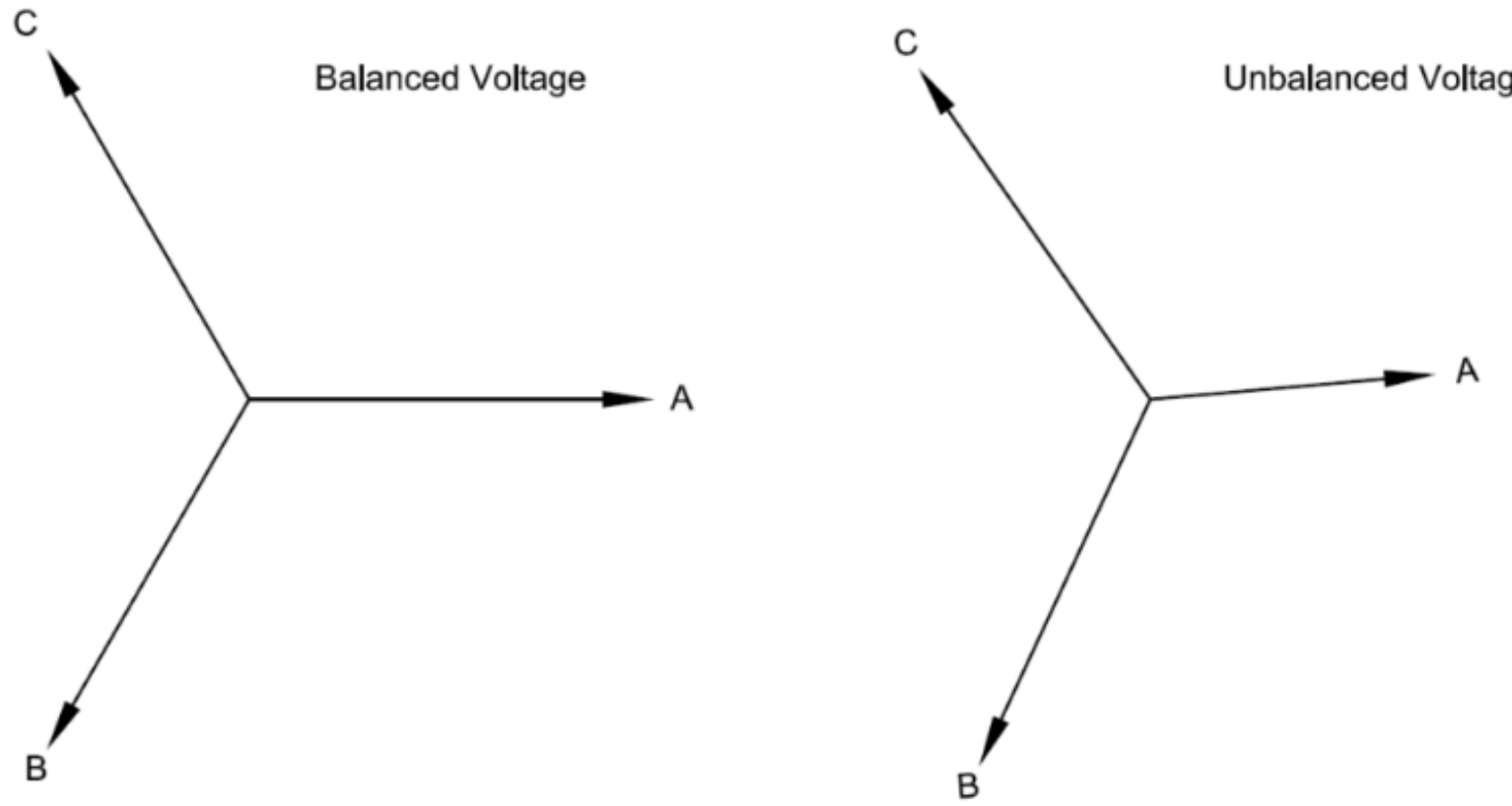

Figure 1 Unbalanced Voltage

Three phase systems could conceivably have an impartial wire. A nonpartisan wire permits the three phase system to utilize a higher voltage while as yet supporting lower voltage single phase machines. In high voltage appropriation circumstances it is regular not to have a nonpartisan wire as the voltages can essentially be 
associated between phases (phase-phase association). Three phase has properties that make it entirely alluring in electric power systems. To start with, the phase currents will in general offset each other, adding to focus on account of a direct adjusted voltage. This makes it conceivable to take out the impartial conductor on certain lines; all the phase conductors convey a similar current thus can be a similar size, for a fair voltage. Second, power move into a straight adjusted voltage is steady, which assists with lessening generator and engine vibrations. At long last, three-phase systems can create an attractive field that pivots a predetermined way, which disentangles the plan of electric engines. Three is the most reduced phase request to show these properties. Most homegrown voltages are single phase. By and large three phase power either doesn't go into homegrown houses by any stretch of the imagination, or where it does, it is part out at the fundamental dispersion board.

At the power station, an electrical generator changes over mechanical power into a bunch of alternating electric currents, one from each electromagnetic loop or twisting of the generator. The currents are sinusoidal elements of time, all at similar frequency however balance so as to give various phases. In a three-phase system the phases are divided similarly, giving a phase division of 33\% cycle. The power frequency is regularly $50 \mathrm{~Hz}$ in Asia, Europe, South America and Australia, and $60 \mathrm{~Hz}$ in the US and Canada (however observe Mains power systems for more detail). Generators output at a voltage that goes from many volts to 30,000 volts. At the power station, transformers "venture up" this voltage to one more reasonable for transmission. After various further transformations in the transmission and conveyance network the power is at last changed to the standard mains voltage (for example the "family unit" voltage). The power may as of now have been part into single phase now or it might even now be three phase.

Where the stepdown is 3 phase, the output of this transformer is typically star associated with the standard mains voltage (120 V in North America and $230 \mathrm{~V}$ in Europe and Australia) being the phase-impartial voltage. Another system regularly found in North America is to have a delta associated auxiliary with a middle tap on one of the windings providing the ground and impartial. This takes into account $240 \mathrm{~V}$ three phase just as three distinctive single phase voltages ( $120 \mathrm{~V}$ between two of the phases and the impartial, $208 \mathrm{~V}$ between the third phase (known as a high leg) and nonpartisan and $240 \mathrm{~V}$ between any two phases) to be made accessible from a similar stock. In electricity, the phase alludes to the appropriation of a load. What is the contrast between single-phase and threephase power supplies? Single-phase power is a two-wire alternating current (ac) power circuit. Ordinarily, there is one power wire - the phase wire and one nonpartisan wire, with current streaming between the power wire (through the load) and the unbiased wire. Three-phase power is a three-wire AC power circuit with each phase ac signal 120 electrical degrees separated. Private homes are typically served by a solitary phase power supply, while business and mechanical offices as a rule utilize a three-phase supply. One key distinction between singlephase versus three-phase is that a three-phase power supply better obliges higher loads.

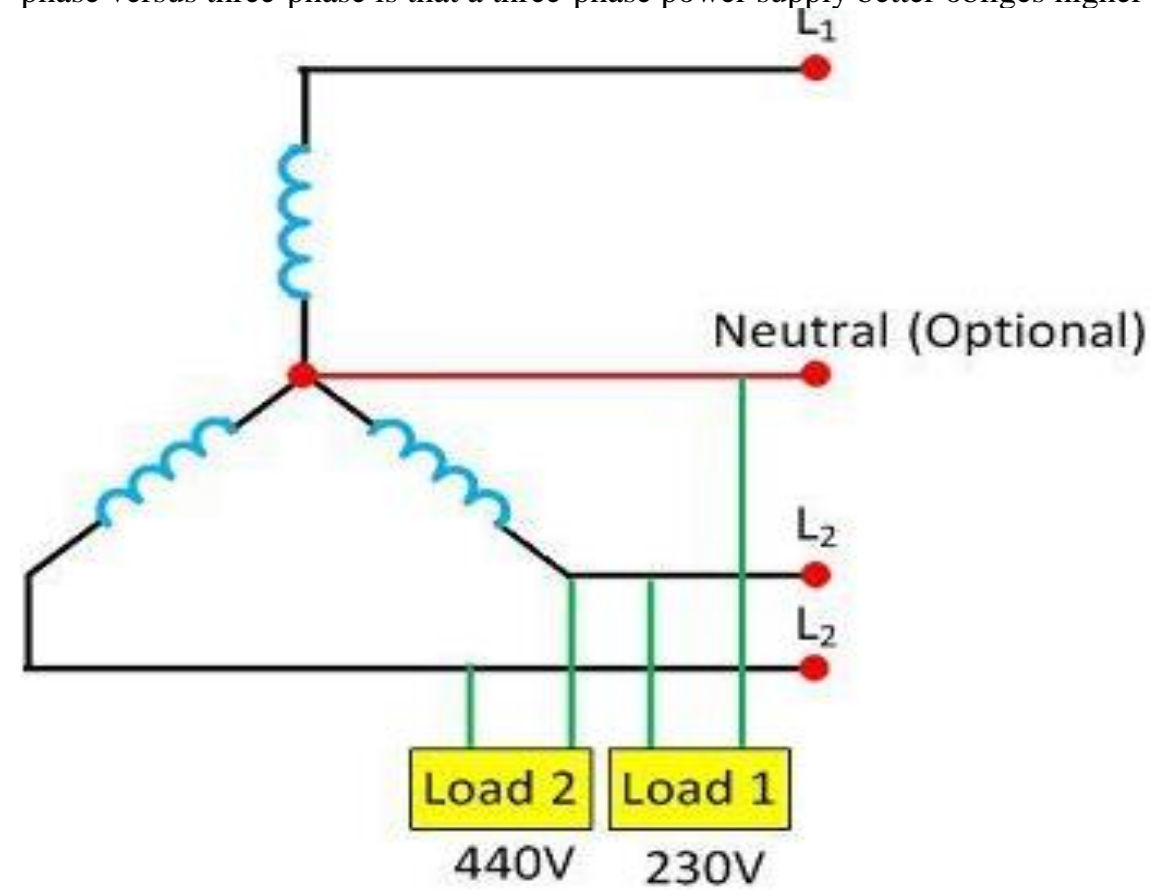

Figure 2 Three Phase Connection

Single-phase power supplies are most usually utilized when regular loads are lighting or warming, as opposed to huge electric engines. Single-phase systems can be gotten from three-phase systems. In the US, this is done by means of a transformer to get the legitimate voltage, while in the EU it is done straightforwardly. Voltage levels in the EU are with the end goal that a three-phase system can likewise fill in as three single-phase systems. One 
other significant distinction between 3-phase powers versus single phase power is the consistency of the conveyance of power. Due to the pinnacles and plunges in voltage, a solitary phase power supply essentially doesn't offer a similar consistency as a three-phase power supply. A three-phase power supply conveys power at a consistent, steady rate. Looking at single-phase versus three-phase power, three-phase power supplies are more proficient. A three-phase power supply can communicate three fold the amount of power as a solitary phase power supply, while just requiring oneextra wire (that is, three wires rather than two). In this way, three-phase power supplies, regardless of whether they have three wires or four, utilize less conduit material to send a set measure of electrical power than do single-phase power supplies. Some three-phase power supplies do utilize a fourth wire, which is a nonpartisan wire. The two most basic setups of three-phase systems are known as wye and delta. A delta design has just three wires, while a wye arrangement may have a fourth, nonpartisan, wire. Single-phase power supplies have a nonpartisan wire also. Both single-phase and three-phase power conveyance systems have parts for which they are appropriate. In any case, the two sorts of systems are very unique in relation to one another.

\section{Literature Review}

Vicente León-Martínez (2020): Similarly to how Steinmetz built up his hypothesis of alternating current in single-phase sinusoidal systems, a couple of formal connections between articulations of the instantaneous and Buchholz's clear power in three-phase systems were distinguished in this paper. In light of these connections, a procedure to communicate Buchholz's obvious power and its segments in any three-phase, wye-arranged system sinusoidal or non-sinusoidal, adjusted or unbalanced through instantaneous power articulations was set up. The utilization of the proposed strategy to the whole system permitted the assurance of a novel amount alluded to as nonpartisan dislodging power, which estimated the effects of the marvels brought about by the impartial way procedure on the estimations of the source and load obvious power. These effects were examined utilizing a certifiable metropolitan establishment with an unbiased conductor decay reenactment by means of an Excel stage as an application model.

RyutoShigenobu (2020): This paper presents a compensation technique for unbalanced voltage through dynamic and responsive power control by using a keen inverter that improves the voltage unbalance record and distinguishes an unbalanced condition of voltage greatness and phase, and hence upgrades power quality by limiting the voltage lopsidedness. Above all else, this paper presents an investigation of a numerical methodology, which shows that the ordinary voltage unbalanced factor (VUF) utilizing the even part can't effectively identify the imbalanced state from list conditions; and by just limiting the VUF esteem, it can't set up a decent condition for an unbalanced condition of the voltage profile. This paper further examines that discontinuous photovoltaic (PV) output power and expanded load request lead to a startling voltage unevenness. Consequently, considering the unpredictability of unbalanced voltage conditions, a particular load and a PV profile were separated from large information and applied to the circulation system model. The adequacy of the proposed plot was confirmed by contrasting VUF files and controlling the dynamic and receptive power of a shrewd inverter through a mathematical recreation.

Blasco (2019): The compensation of an electrical system from latent compensators primarily centers on direct systems where the utilization of charges doesn't change essentially over the long run. In three-phase three-wire systems, when the organization voltages are unbalanced, negative-succession voltages and currents show up, which can fundamentally build the absolute evident power provided by the organization. This likewise builds the organization misfortunes. This paper presents a technique for figuring the compensation of the positivesuccession responsive power and unbalanced powers brought about by the negative-arrangement line currents utilizing receptive components (loops as well as capacitors). The compensation is applied to three-phase threewire straight systems with unbalanced voltages and loads, which are associated with a boundless power organization. The strategy is autonomous of the load attributes, where just the line-to-line voltages and line currents, at where compensation is wanted, should be known ahead of time. The arrangement acquired is ideal, and the system saw from the organization carries on as one that just burns-through the dynamic power needed by a load with a completely adjusted current system. To comprehend the proposed technique and show its legitimacy, a contextual analysis of a three-phase three-wire straight system associated with an endless power network with unbalanced voltages and currents is directed.

\section{PROPOSED METHODOLOGY}

This system comprises of a plant (transport B2), reproduced by a $1 \mathrm{MW}$ resistive load and an engine load (ASM) took care of at $2400 \mathrm{~V}$ from a dissemination $25 \mathrm{kV}$ system through a $6 \mathrm{MVA}, 25 / 2.4 \mathrm{kV}$ transformer, and from a crisis coordinated generator/diesel motor unit (SM). The $25 \mathrm{kV}$ system is demonstrated by a basic R-L equal source (impede $1000 \mathrm{MVA}$, quality factor $\mathrm{X} / \mathrm{R}=10$ ) and a $5 \mathrm{MW}$ load. The offbeat engine is evaluated 2250 $\mathrm{HP}, 2.4 \mathrm{kV}$, and the coordinated machine is appraised 3.125 MVA, $2.4 \mathrm{kV}$. At first, the engine builds up a mechanical power of $2000 \mathrm{HP}$ and the diesel generator is in backup, conveying no dynamic power. The coordinated machine thusly works as a simultaneous condenser creating just the receptive power needed to control the $2400 \mathrm{~V}$ transport B2 voltage at $1.0 \mathrm{pu}$. At $\mathrm{t}=0.1 \mathrm{~s}$, a three-phase to ground shortcoming happens on 
the $25 \mathrm{kV}$ system, causing the kickoff of the $25 \mathrm{kV}$ circuit breaker at $\mathrm{t}=0.2 \mathrm{~s}$, and an unexpected increment of the generator loading. During the transient period following the issue and islanding of the engine generator system, the coordinated machine excitation system and the diesel speed lead representative respond to keep up the voltage and speed at a steady worth.

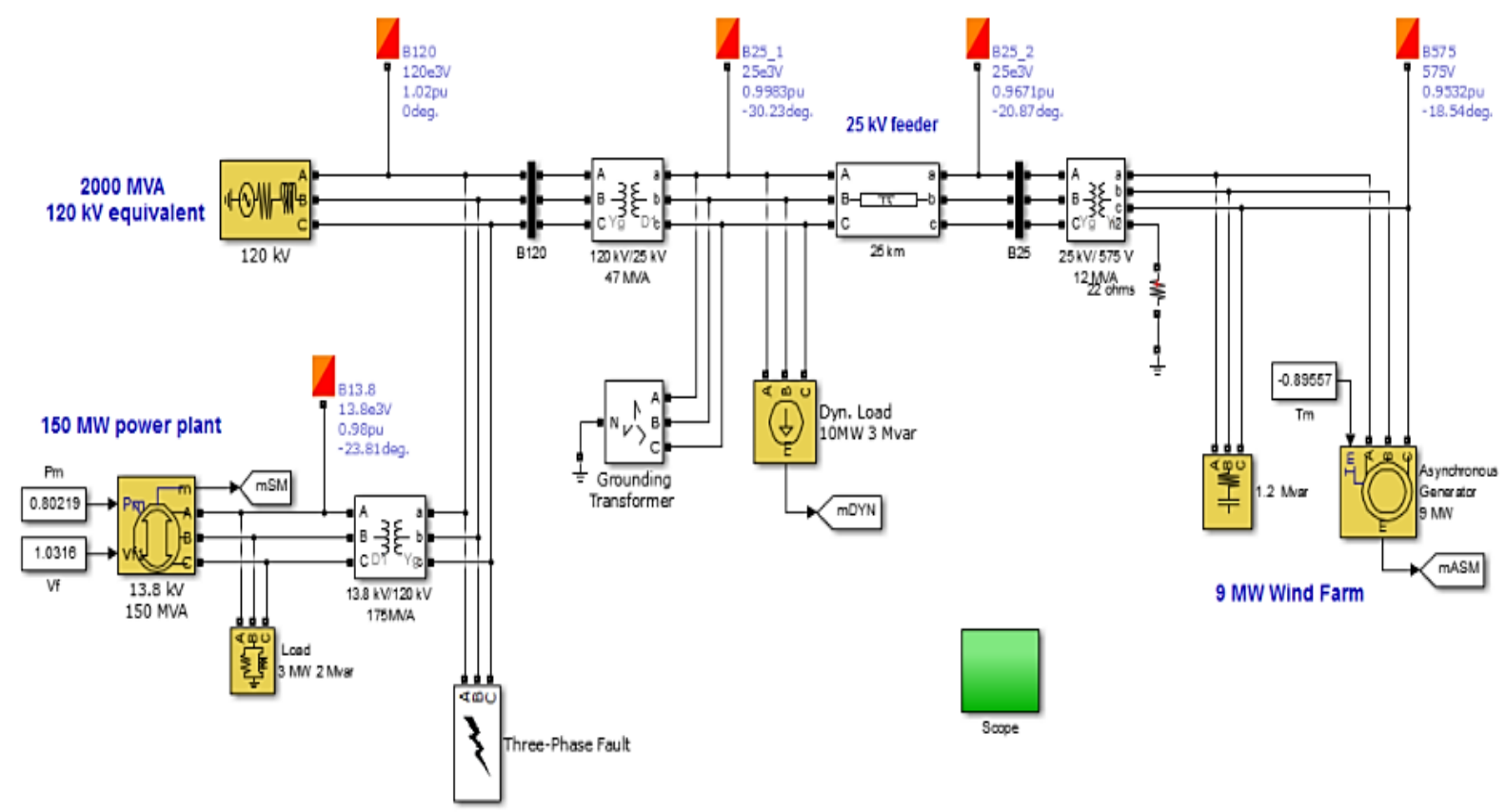

Figure 3 Simulink Circuit

These underlying conditions are SM block: Initial estimations of speed deviation (typically 0\%), rotor point, extents and phases of currents in stator windings, and beginning field voltage needed to acquire the ideal terminal voltage under the predefined load stream ASM block: Initial estimations of slip, rotor point, sizes and phases of currents in stator windings. Open the discourse box of the Synchronous Machine and Asynchronous Machine blocks. All underlying conditions should be set at 0, aside from the underlying SM field voltage and ASM slip, which are set at $1 \mathrm{pu}$. Open the three extensions checking the SM and ASM flags just as the transport B2 voltage. Start the recreation and notice the initial $100 \mathrm{~ms}$ before deficiency is applied. As the recreation begins, note that the three ASM currents start from zero and contain a gradually rotting DC segment.

When playing out a load stream investigation, you may have to emphasize on $\mathrm{P}, \mathrm{Q}, \mathrm{V}$ qualities until you find good voltages at all transports. This may need, for instance, changing created power, load powers, or responsive shunt compensation. To change the load stream arrangement, you need to alter the boundaries of the load stream blocks and of the Load Flow Bus blocks. At that point click the Update catch to revive the load stream information showed by the table. The past load stream arrangement is then erased from the table. Snap the Compute catch to get another load stream arrangement comparing to the progressions you made. Whenever you have gotten a good load stream, you need to refresh the model beginning conditions as per the load stream arrangement.

Snap the Apply to Model catch to instate the machine squares of the model, just as the underlying states of controllers associated with the machines. Open the Three-Phase Parallel RLC Load block associated at the B13.8 transport. As the Load type determined in the Load Flow tab is steady PQ, the ostensible voltage of this square has been changed to the relating transport voltage of $0.98 \mathrm{pu}$. The Nominal phase-phase voltage boundary is set to $(13800) * 0.98$. Open the Three-Phase Dynamic Load block associated at the B25_1bus. The Initial positivearrangement voltage VO is set to [0.998241 pu - $30.2228 \mathrm{deg}$ ]. Note that the voltage extents and points acquired at each transport have been composed as square comments under the Load Flow Bus blocks.

- Open the degree and start the reproduction.

- The Three-Phase Fault block has been modified to apply a six-cycle flaw at B120 transport.

- Observe waveforms of SM dynamic power, SM and ASM rates, and PQ of DYN load, and notice that recreation begins in consistent state.

The machine speeds set aside any longer effort to balance out due to the latency of the engine/load and diesel/generator systems. In our model, the ASM even begins to pivot off course on the grounds that the engine turning over force is lower than the applied load force. Stop the reproduction. The load stream boundaries in the Preferences tab of the Powerful are utilized to fabricate the Ybus network permission framework and to settle the 
load stream. The base power is utilized to determine units of the standardized Ybus lattice in pu/Pbase and transport base voltages. The power_LFnetwork_5bus model contains five transports; thusly, the Ybus framework will be a $5 \times 5$ complex network assessed at the frequency indicated by the Frequency (Hz) boundary. The load stream calculation utilizes an iterative arrangement dependent on the Newton-Raphson strategy. The Max cycle's boundary characterizes the most extreme number of emphases. The load stream calculation will repeat until the $\mathrm{P}$ and Q bungle at each transport is lower than the PQ resilience boundary (in pu/Phase). The power befuddle is characterized as the contrast between the net power infused into the transport by generators and PQ loads and the power communicated on all connections leaving that transport. To evade a seriously adapted Ybus lattice, you should choose the Base power boundary esteem in the scope of ostensible powers and loads associated with the organization. For a transmission network with voltages going from $120 \mathrm{kV}$ to $765 \mathrm{kV}$, a 100 MVA base is normally chosen. For a dissemination organization or for a little plant comprising of generators, engines, and loads having an ostensible power in the scope of many kilowatts, a 1 MVA power base is better adjusted.

\section{EXPERIMENT RESULT}

In the wake of getting a power supply on a $220 \mathrm{v} A C$ is initial changed over into a $12 \mathrm{vAC}$ with the assistance of focus tap stepdown transformer, as the majority of the control and observing circuit requires a different DC Sources, consequently the $12 \mathrm{vAC}$ at first changed over into $12 \mathrm{vDC}$ with the assistance of Rectifier and Filter made by utilizing Diode D1, D2 and C1 of 1000uF. The principle point of circuit is to distinguish a Sag and Swell in input voltage and further it is amended and make consistent in output with some resistance of $(+/-5 \%)$. As we can Boost the voltage by some percent with the assistance of capacitor in the event of Sag in Input voltage, however they helped voltage can't be controlled, Hence a functioning reactor is utilized, which can increment just as diminishes the voltage by controlling an Input voltage providing for it. In this circuit a functioning reactor can help a voltage to $220 \mathrm{v}$ by giving a base voltage of $140 \mathrm{v}$, and can support a voltage up to $280 \mathrm{v}$ by giving a base voltage of $220 \mathrm{v}$. Microcontroller PIC is utilized in this circuit which requires a consistent $5 \mathrm{vDC}$. To change a $12 \mathrm{vDC}$ over to $5 \mathrm{vDC}$, a Regulator IC 7805 is utilized, which keeps a 5vDC consistent regardless of any variances between $6 \mathrm{v}$ to $28 \mathrm{v}$. At first PIC checks the Input voltage \& Output voltage. As the input and output voltage is enormous and past the estimating furthest reaches of PIC, for example 5vDC, henceforth a PT of $220 \mathrm{v} / 6 \mathrm{v}$ is utilized. The prerequisite of PIC for estimation is $0-5 \mathrm{vdc}$, consequently the output of PT additionally changed over in a DC with the assistance of another rectifier and channel.

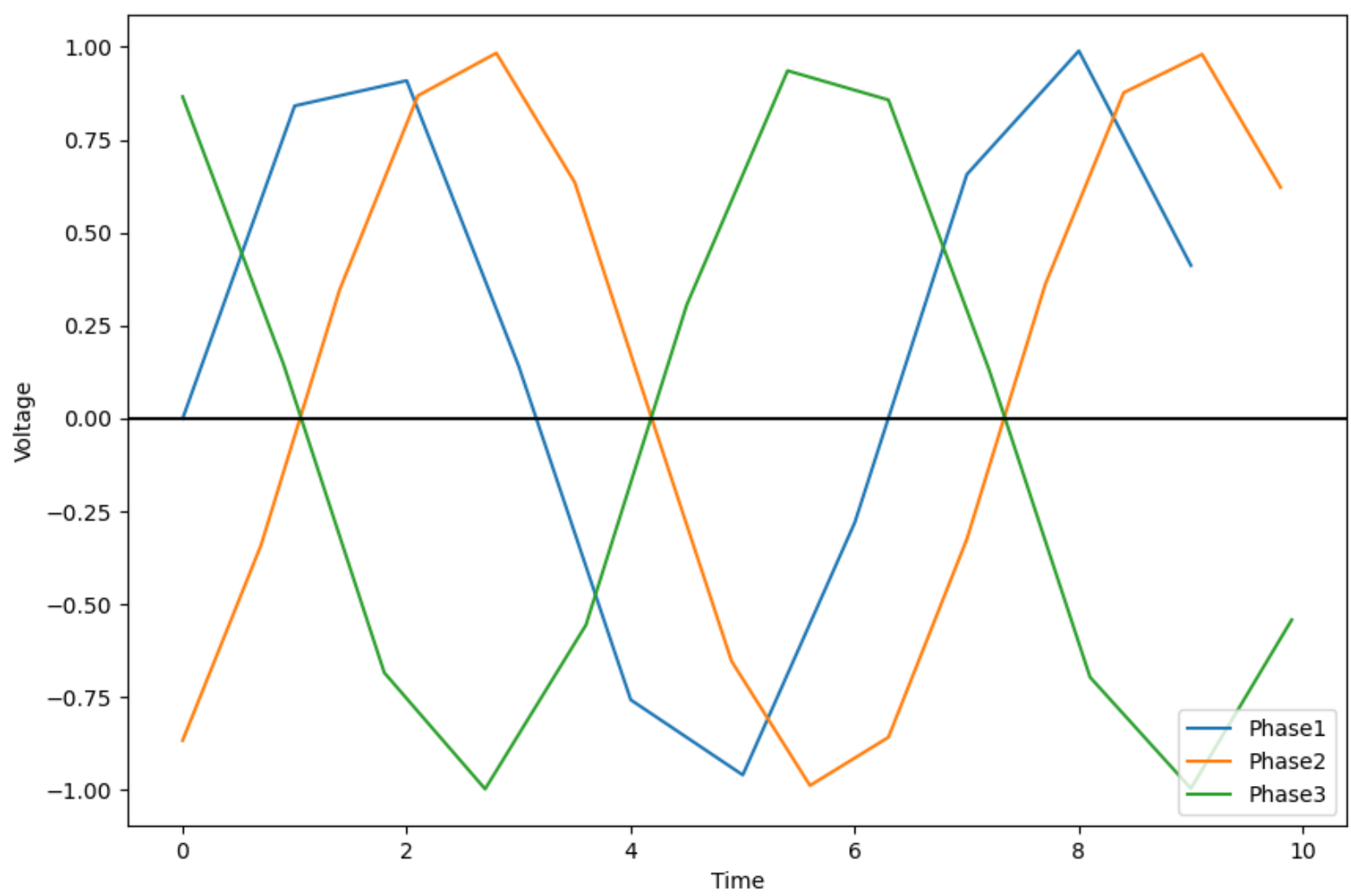

Figure 4 Unbalance Voltage 


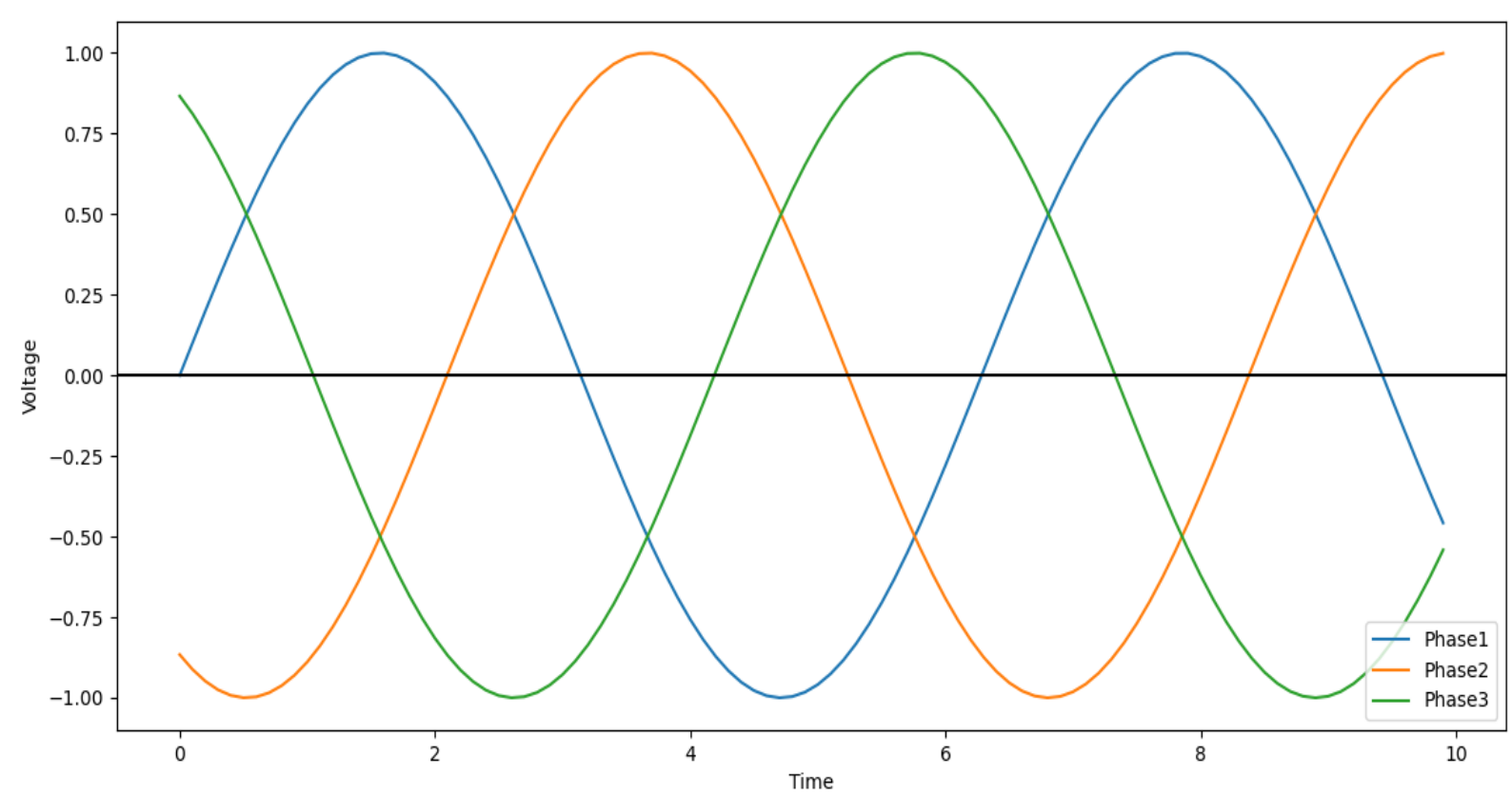

\section{CONCLUSION}

Figure 5 Balanced Voltage

In this paper we have actualized instantaneous power hypothesis for unbalanced voltage compensation of 3phase power systems. Power hypothesis is equipped for remunerating the different voltage unsettling influences like single phase and two phase list and swell in unbalanced condition just as hang and swell in unbalanced condition in three phases. Likewise we have demonstrated voltage compensation for three phase power system utilizing MATLAB Simulink test system instrument. Different conditions are tried for the exhibition capacity of power hypothesis through broad reproduction and results are confirmed. From the outcome we can reason that voltage flimsiness was inspected and wiped out insecurity utilizing power hypothesis idea. Thus we have accomplished adjusted voltage solidness from unbalanced voltage compensation of 3-phase power systems. Power hypothesis is tried for adjusted droop, swell, various list and numerous swell and list and swell cases, and in unbalanced condition hang and swell in single and two phases just as unbalanced three phase condition.

\section{REFERENCES}

1. Blasco 2019 "Compensation of Reactive Power and Unbalanced Power in Three-Phase Three-Wire Systems Connected to an Infinite Power Network" DOI: 10.3390/app10010113

2. Vicente León-Martínez 2020 "Relationship between Buchholz's Apparent Power and Instantaneous Power in Three-Phase Systems" doi:10.3390/app10051798

3. RyutoShigenobu 2020 "Optimization of Voltage Unbalance Compensation by Smart Inverter" doi:10.3390/en13184623

4. Nour, A.M. Review on voltage-violation mitigation techniques of distribution networks with distributed rooftop PV systems. IET Gener. Transm. Distrib. 2020, 14, 349-361.

5. Iioka, D.; Fujii, T.; Orihara, D.; Tanaka, T.; Harimoto, T.; Shimada, A.; Goto, T.; Kubuki, M. Voltage reduction due to reverse power flow in distribution feeder with photovoltaic system. Int. J. Electr. Power Energy Syst.2019, 113, 411-418.

6. El-Hawary, M.E. Definitions of Voltage Unbalance. IEEE Power Eng. Rev. 2001, 21, 49-51.

7. Zaheb, H.; Danish, M.S.S.; Senjyu, T.; Ahmadi, M.; Nazari, A.M.; Wali, M.; Khosravy, M.; Mandal, P.A Contemporary Novel Classification of Voltage Stability Indices. Appl. Sci. 2020, 10, 1639.

8. Ziadi, Z.; Oshiro, M.; Senjyu, T.; Yona, A.; Urasaki, N.; Funabashi, T.; Kim, C. Optimal Voltage Control UsingInverters Interfaced With PV Systems Considering Forecast Error in a Distribution System. IEEE Trans.Sustain. Energy 2014, 5, 682-690.

9. Dao, V.T.; Ishii, H.; Hayashi, Y. Optimal smart functions of large-scale PV inverters in distributionsystems. In Proceedings of the 2017 IEEE Innovative Smart Grid Technologies-Asia (ISGTAsia), Auckland,New Zealand, 4-7 December 2017; pp. 1-7.

10. Adewuyi, O.B.; Ahmadi, M.; Olaniyi, I.O.; Senjyu, T.; Olowu, T.O.; Mandal, P. Voltage SecurityConstrainedOptimal Generation Rescheduling for Available Transfer Capacity Enhancement in Deregulated ElectricityMarkets. Energies 2019, 12, 4371. 
11. Brown, R.E.; Pinkerton, R. Distribution Reliability Optimization Using Synthetic Feeders. Energies 2019, 12, 3510.

12. Lee, H.J.; Yoon, K.H.; Shin, J.W.; Kim, J.C.; Cho, S.M. Optimal Parameters of Volt-Var Function in SmartInverters for Improving System Performance. Energies 2020, 13, 2294. 